\title{
The 3rd Annual Meeting of The Mountain West Society of Plastic Surgeons
}

\author{
Jackson Hole, WY; March 2018; Published: 28 Feb 2018 \\ Correspondence to: Dr. Raman C. Mahabir, Mayo Clinic Arizona, 5777 East Mayo Blvd, Phoenix, AZ 85054, USA. \\ E-mail: Mahabir.Raman@mayo.edu
}

\section{Pedicled omentum for coverage of extra-abdominal vascular bypass graft in the groin: a case-report}

Neal Moores, Christopher Pannucci

University of Utah

Salvage of infected vascular bypass grafts continues to present one of the most complex reconstructive algorithms for both vascular, and plastic surgeons. Graft infection necessitates graft explantation and extra-anatomic bypass, most often with additional synthetic material. These multiply comorbid patients suffer long periods of convalescence in the ICU with attendant bed-rest, ventilator dependency and sepsis. The omentum is an established and effective source of intra-abdominal coverage for gastrointestinal and urological anastomoses, as well as vascular graft coverage. The omentum is also an established method for coverage of extra-abdominal pathology and as a free flap it may be used in any location. However, its use as a pedicled flap for extra-abdominal reconstruction has hitherto been largely limited to chest wall reconstruction.

\section{Reconstructive phalloplasty aesthetic ideals}

\section{Julia Cook}

Indiana University Division of Plastic \& Reconstructive Surgery

Aim: Aesthetic ideals are often described to guide reconstruction. The number of phalloplasties performed in the United States is steadily increasing; however, there is a paucity of published literature describing the 
reconstructive ideals of the phallus. The purpose of this study was to determine the preferred aesthetics of phalloplasties.

Methods: Phalloplasty aesthetic subunits were defined, and subunit measurements and ratios were examined. A split-testing questionnaire was distributed to medical personnel and online forum users to determine the preferred aesthetic ideals based on phallic subunits. Digitally altered photos focusing on varying ratios of the shaft, glans, corona, and frenulum were presented to participants who were instructed to choose the image that was most aesthetically pleasing. Respondent demographics were collected, including age, sex, medical background, and sexual orientation.

Results: One hundred thirty-nine people completed the survey. The overall preferred aesthetic ratios are as follows: $74.8 \%(n=104)$ preferred a corona length to width ratio of $20: 1,95.7 \%(n=133)$ preferred a shaft length to width ratio of 2.5:1, 51.1\% $(n=71)$ preferred a unilateral taper to the glans, $79.9 \%(n=111)$ preferred no degree of shaft angulation, $69.1 \%(n=96)$ preferred a length to width ratio of $1: 1,56.1 \%(n=78)$ preferred angulation at the glans corona junction, $57.6 \%(n=80)$ preferred a curved curvature to the corona, and $66.2 \%$ $(n=92)$ preferred a straight frenulum.

Conclusion: The preferred aesthetics of the reconstructed phallus varies by individual; however, this study can be a guide for both patients and reconstructive surgeons during surgical planning.

\section{The impact of traumatic dog bite injuries necessitating surgical treatment in elderly}

\section{Tyler Evans}

\section{Indiana University Division of Plastic \& Reconstructive Surgery}

Aim: Traumatic dog bite injuries are a common reason for emergency department visits and hospital admissions. Most literature targets dog bite injuries in pediatric patients, but minimal studies are dedicated to the elderly. This study was designed to investigate the epidemiology and overall healthcare burden of dog bites in the elderly population.

Methods: Data was obtained using the Nationwide Emergency Department Sample (NEDS) database. Inclusion criteria included patients 65 years or older with ICD-9 E90.60=dog bite. Site of injury and cellulitis incidence were obtained. Types of procedures were categorized into two groups: minor and major therapeutic. Inpatient length of stay and total charges were also collected.

Results: A total of 318,161 dog bite ED visits were identified, of which 24,541 were 65 years and older. Over $95 \%$ of patients had only a single injury site, most commonly the upper extremity (72.5\%). The incidence of cellulitis was 7.9\%. Admitted elderly patients often had subsequent therapeutic procedures (minor 40.9\%, major 26.3\%, flap/graft 3.6\%). Average inpatient length of stay was 4 days. The average total charge for ED services only was $\$ 1094$ per patient and combined ED and inpatient services was $\$ 24,551$.

Conclusion: Dog bite-related injuries are a significant source of trauma presenting to ED and often requiring surgical treatment and hospital admissions, especially in elderly. The impact of dog bite injuries poses a substantial burden on public health both medically and financially. Increased vigilance in prevention of these injuries and their associated complications is crucial to reduce overall morbidity and medical costs. 


\section{When free tissue transfer is not an option: complex traumatic lower extremity reconstruction using perforator flaps}

\section{Ryan Kunkel, Christopher Demas}

\section{University of New Mexico Hospital}

Perforator flaps have changed the approach to reconstructive dilemmas since their emergence on the plastic surgery scene. This additional rung on the reconstructive ladder has led to new methods of closing complicated lower extremity wounds. While arguably more versatile than traditional methods of lower extremity reconstruction, they require tedious dissection and microsurgical techniques. It is important for the plastic surgeon to be familiar with the application of perforator flaps, particularly when more traditional methods of reconstruction are not viable or safe options. We present three cases of complicated lower extremity wounds that were reconstructed with perforator flaps. The first case was a Grade IIIB tibia with a $22-\mathrm{cm}$ soft tissue defect involving all three zones of the lower extremity. Renal and hepatic failure precluded a prolonged microsurgical procedure. This was covered with a distally based posterior tibial perforator flap and a gastrocnemius flap. The second case was a Grade IIIB tibia with open wound in the proximal and middle third of the leg. The patient failed gastrocnemius and reverse sural flap by another surgeon and was not a candidate for a free flap because of a recent STEMI. He was successfully reconstructed with two flaps based on one posterior tibial perforator cluster. The third case was a grade IIIC tibia with 12$\mathrm{cm}$ segmental bone loss. Free tissue transfer was too risky because of a femoral vein thrombosis, which, if propagated, would cause flap failure. Double opposing peroneal and posterior tibial perforator flaps were used to successfully close the defect.

\section{Oncoplastic nipple sparing mastectomy with immediate, implant-based reconstruction: technique and outcomes}

Sarah E. Sasor, Julia A. Cook, Tyler A. Evans, William A. Wooden, Sunil S.Tholpady, Michael W. Chu, Juan Socas

Indiana University Division of Plastic \& Reconstructive Surgery

Aim: Nipple sparing mastectomy (NSM) is a popular option for women with small, peripherally located breast tumors. The procedure is oncologically safe in select patients but can be technically challenging in large, ptotic breasts. In this study, we examine our experience with NSM using a Wise-pattern skin reduction and nipple areola complex (NAC) preservation on an inferiorly based adipo-dermal pedicle.

Methods: A retrospective study of patients undergoing NSM at our institution over a six month period was performed. All patients undergoing NSM with a Wise-pattern skin reduction, NAC repositioning, and immediate, implant-based breast reconstruction were included. Variables collected included patient demographics, smoking status, medical comorbidities and indication for mastectomy. Post-operative outcomes were analyzed with respect to patient and tumor characteristics.

Results: Eight NSM's were performed on five patients during the study period. Mean age and BMI were 49 years and 29.1, respectively. No patients were current smokers. All breasts had grade 2 or 3 ptosis. Mean mastectomy weight was 878 grams. All patients had immediate reconstruction with 
tissue expander (6) or implant (2) placement. One patient required take-back to the operating room for hematoma evacuation. Three NAC's demonstrated partial necrosis; all were successfully treated with local wound care. No NAC loss, T-junction breakdown, wound dehiscence, infection or implant extrusion/exposure was noted.

Conclusion: Wise-pattern skin incision with NAC repositioning and immediate, implant-based reconstruction is a safe and useful technique for managing large, ptotic breasts during NSM. In carefully selected patients, it has a high success rate and provides optimal aesthetic results.

\title{
6. Cherubism in a 4-year-old boy managed with tumor debulking and mandibular osteotomies and repositioning
}

\author{
Rhett Willis, Jared Garlick, Daniel Donato, Barbu Gociman
}

The University of Utah

Cherubism is a hereditary, disfiguring growth of the mandibles/maxilla in young children. It is characterized by significant loss of medullary bone which is replaced by excessive amounts of fibrous tissue. It is an extremely rare (300 reported cases), autosomal dominant disease. Although generally self-limiting, when severe, can cause significant functional and psychosocial impairment for the patient. Current literature advocates a "wait and watch approach", as majority of these will partially or completely remit after puberty. A 4-year old boy presented with rapidly enlarging mandible and maxilla causing significant change in the facial contour, malocclusion and phonation difficulties. He was treated with aggressive tumor debulking. In addition, using a piezo-electric saw, the lateral cortexes of the mandibular rami, angles and bodies were osteotomized and repositioned bilaterally. This allowed the obliteration of the grotesquely enlarge medullary spaces and restoration of a normal mandibular anatomy. At 8 months postoperatively, the patient had significant improvement in facial contouring and a normal outward appearance and stable dentition. A CT scan showed significant ossification around the molars at the site of mandibular bone repositioning. Although in the majority of cherubism cases there is spontaneous regression after puberty, the sequela of this disfiguring condition in the patient's formative years can cause a lifetime of psychological and physical stress in addition to permanent soft tissue and dental abnormalities. Based on our experience, we recommend that early surgical intervention in severe cherubism cases be strongly considered.

\section{Beauty and the bull: traumatic rupture of a breast implant in Pamplona, Spain}

\section{Nicole Kurnik, Alanna Rebecca, Lyndsey Bryant}

Mayo Clinic - Arizona

Aim: Bullhorn injuries are more common in Ibero-American countries where bulls are involved in sport. There is a lack of information in the literature regarding these injuries. The purpose of this study is to present a case of a bullhorn injury to the thorax causing a ruptured and infected breast implant and a review the literature. 
Methods: A pub-med literature search was performed and all available English-language publications pertaining to bull injuries during sport were included.

Results: A 54 year-old female sustained a penetrating bullhorn injury to her thorax causing breastimplant rupture and resulting infection presented to our institution. She was treated with broad-spectrum intravenous antibiotics, removal of bilateral breast implants and wound debridement. Intraoperative cultures grew methicillin resistant coagulase-negative staphylococcus. After treatment with Bactrim, her implants were replaced; fat grafting of the contour deformity and pectoralis repair was performed. Upon review of the literature, emergency assistance is required during bull-related sports approximately $9 \%$ of the time. Body parts most commonly affected are the extremities (66\%), inguinal/perineal regions (19\%-54\%) thorax $(3 \%-10 \%)$ and head and neck (10\%-19\%). Infection occurs in up to $60 \%$ of those injured, yet there is minimal reported on what bacteria is cultured. Staphylococcus epidermidis has been reported and also occurred in our patient.

Conclusion: Bullhorn injuries can result in devastating injuries with high infection risk which go beyond typical penetrating trauma. To our knowledge this is the first reported case of a breast implant rupture with subsequent infection due to a bullhorn injury.

\title{
8. Transparency of provider education and board certification among cosmetic surgeons
}

\author{
Jared Garlick, Kristofor Olson, Madison Hunt, Daniel Donato, Christopher Pannucci, Courtney
} Crombie

\section{University of Utah}

Aim: The number of non-plastic surgery trained providers offering cosmetic procedures in the U.S. is increasing. Utah's Division of Occupational \& Professional Licensing currently has no way of tracking the number of providers offering cosmetic procedures. The purpose of this study was to analyze providers throughout Utah who offer cosmetic procedures.

Methods: Providers in Utah who offered at least one of three cosmetic procedures (breast augmentation, liposuction, and Botox) were included in the study. Formal medical training, board certifications, marketing practices, and procedures offered outside of scope of practice were extracted from publicly available provider websites. All data was analyzed by specialty and Chi-square analyses were performed comparing the categorical data.

Results: Nineteen different medical specialties throughout Utah were identified. Nearly one in five providers offering breast augmentation are non-plastic surgery trained providers. Only 50\% of providers offering liposuction are trained plastic surgeons, and $75 \%$ of Botox providers are not plastic surgeons. Regarding breast augmentation, liposuction, and Botox, plastic surgeons are more likely than non-plastic surgery providers to list their education ( $93 \%$ vs. $42 \%, P=0.037$; $93 \%$ vs. 53\%, $P<0.001$; $97 \%$ vs. $55 \%, P<0.001$; respectively) and board certifications ( $90 \%$ vs. $25 \%, P=0.007 ; 90 \%$ vs. $40 \%, P<0.001$; $95 \%$ vs. $48 \%, P<0.001$; respectively) on their website.

Conclusion: We draw attention to this disparity in provider training and marketing practices, while highlighting a need for increased transparency of cosmetic provider credentials as a way to educate and potentially increase patient safety. 
9. Alloplastic cranioplasty reconstruction: a systematic review comparing outcomes with titanium mesh, polymethyl methacrylate, polyether ether ketone, and norion implants

\author{
Jeremie Oliver, Joseph Banuelos Mancilla, Krishna Vyas, Basel Sharaf
}

Mayo Clinic - MN

Aim: Alloplastic cranioplasty has evolved significantly over the years with the development of different materials to serve as a medium of repair to the defect, such as titanium mesh (Ti), polymethyl methacrylate (PMMA), polyether ether ketone (PEEK), and norion implants. There has yet to be published a systematic review of such outcomes among the alloplastic materials we have compared in this study. Our objective in this study was to compare postoperative rates of infection, local complications and allograft failures following cranioplasty reconstruction using Ti, PMMA, PEEK, and norion implants.

Methods: Newcastle-Ottawa Quality Assessment Scale guidelines were used for article identification, screening, eligibility and inclusion. The electronic literature search included Medline/Pubmed, Scopus and Cochrane Database.

Results: Eighty-three studies and 5320 patients (mean age $=40.6$ years) were included in our review $(\mathrm{Ti}=2383$, PMMA $=2116, \mathrm{PEEK}=487$, norion $=334)$. Overall, $\mathrm{Ti}$ was associated with the lowest post-operative infection rate $(4.91 \%, P=0.0026$, Pearson's Exact Test) compared to all other sub-groups. PMMA implants were associated with the highest infection rate $(7.23 \%, P=0.0021$, Pearson's Exact Test). Norion implants were associated with the highest local complication rate (15.27\%) but this was not statistically significant. PEEK implants were associated with the highest graft failure rate (6.78\%) although this was only slightly higher than other implant types and did not show statistical significance.

Conclusion: This preliminary analysis begins to address the knowledge gap in determining the infection, local surgical complication and failure rates in alloplastic cranioplasty procedures, although longer-term and randomized trials are warranted to validate any association found in this study.

\title{
10. A single center retrospective evaluation of a surgical strategy to battle biofilm utilizing absorbable antibiotic beads
}

Joseph Gorvetzian, Christopher Demas, Ryan Kunkel

\section{University of New Mexico School of Medicine}

Aim: The implications of surgical site infections are undeniable. Increasingly, biofilms are being recognized as potent adversaries that promote wound infection, complicate healing, and resist attempts at treatment. Targeted delivery of ultrahigh concentrations of antibiotics following wound debridement may present a means of mitigating biofilm establishment while simultaneously minimizing the unsavory side effects of high dose systemic antibiotics. This study aimed to present a case series evaluating an antibiotic bead-based method for addressing these problematic wounds.

Methods: A retrospective analysis of 83 surgeries on 60 patients with high susceptibility to biofilm infection utilizing a strategy of debridement and absorbable antibiotic-laden calcium sulfate bead placement was 
conducted. The surgeries consisted of complex wound and breast reconstruction performed by the senior author (C.P.D.) over 4 years at a single institution. Rates of infection, readmission, and reoperation in the 30-day postoperative period were collected.

Results: Of the 83 cases, there were two instances of subsequent surgical site infection (2.4\%). A total of 16 reoperations were performed (19\%), but in only one case was reoperation required for infectious etiology (1.2\%). Readmission rate following the 83 surgeries was $2.4 \%$. None of the 21 breast-related cases necessitated reoperation or readmission.

Conclusion: These results lend support to the efficacy of absorbable antibiotic-laden beads in delivering supratherapeutic and sustained levels of antibiotics to local areas. Their use in complex and infection-prone wounds may present a valuable addition to the arsenal of plastic and reconstructive surgeons in managing problematic wounds recalcitrant to standard strategies of debridement and systemic antibiotics.

\title{
11. A case report of breast reconstruction with a DCIA-based SIEA free flap
}

\author{
Jonathan Cook, Savannah Moon, Alexander Earle, Miguel Medina
}

\section{Cleveland Clinic Florida}

The superficial inferior epigastric artery (SIEA) flap can be used for autologous breast reconstruction when a muscle sparing operation is desired. Although the deep circumflex iliac artery (DCIA) flap is one of the most commonly used flaps for mandibular reconstruction, to our knowledge, this is the first reported case of a DCIA-based SIEA flap used for breast reconstruction. We report a unique anatomic variant of the origin of a superficially supplied abdominal flap. The patient was a 66-year-old female, with invasive ductal carcinoma of the left breast, and lobular carcinoma in situ of the right breast who underwent bilateral skin sparing mastectomies and immediate autologous reconstruction. The left sided flap demonstrated poor DIEP perforators on pre-operative CTA and was planned as a likely SIEA flap for the left hemi-abdomen. On elevation of the left flap the presumed SIE vessels were identified and traced to the left DCIA system. Clinical perfusion testing and intraoperative ICG fluorescence imaging revealed that the flap was well perfused, and it was used to support the abdominal flap. The flap was transferred with a good vessel size match and no complications. On retrospective analysis this vessel was noted to originate from the DCI system on preoperative CTA. Although the SIEA usually arises from the circumflex femoral artery, its origin from the DCIA represents an unusual anatomical variant, which may have been otherwise overlooked as a dominant perforator. Awareness of this anatomical variant can enable future surgeons to recognize this versatile muscle-sparing alternative.

\section{Work-related physical discomfort in ASCFS and ASMS members: a survey}

\author{
Ashley L. Howarth, Susan Hallbeck, Valerie Lemaine, Davinder J. Singh, Shelley S. Noland
}

Mayo Clinic - AZ

Aim: Risks of physical discomfort and injury are high in cranio/maxillofacial surgeons (CMS), who perform surgeries with headlights and magnification. Identifying the prevalence and impact of work- 
related physical discomfort (WRPD) will guide strategies to prolong surgeon well-being, job satisfaction, and career duration.

Methods: After IRB approval, a 30-question survey was administered to the American Society of Craniofacial Surgery and the American Society of Maxillofacial Surgery members to evaluate surgeons' current physical discomfort. Responses were collected by the Mayo Clinic Survey Center.

Results: Ninty-five respondents, $75 \%$ male, $56 \%$ aged $31-50$ years old, $73 \%$ in academic practice. On a scale of 0-10 (0 no pain, 10 worst pain), WRPD had a median of 3 (surgery without loupes/microscope), 4 (loupe surgery), and 5 (microscope surgery). Pain during, immediately after, and day after surgery was most common in the neck. Pain within $4 \mathrm{~h}$ of surgery was present in $55 \%$. Thirty-eight percent had pain influencing future surgical performance. Operating time was $>6 \mathrm{~h}$ per day (68\%) and $>3$ days per week (72\%). Surgeon discomfort affects posture (72\%), stamina (32\%), sleep (28\%), surgical speed (24\%), relationships (18\%), and concentration (17\%). Twenty-two percent sought medical treatment for discomfort while $9 \%$ took time off work for treatment.

Conclusion: WRPD is a critical issue amongst CMS. Nearly all surveyed experience physical discomfort regularly. This negatively impacts daily life and often requires medical treatment. Thirty-eight percent of respondents felt that WRPD would limit their future careers, perhaps the most concerning finding. It is imperative that CMS employ preventive strategies to combat WRPD.

\section{A2 pulley reconstruction - a novel approach using allograft}

\section{Andrew Peredo, Ashley Ignatiuk}

\section{University of Colorado - Denver}

Flexor tendon pulley injuries are most commonly seen in rock climbers, but reports of ruptures in nonclimbers have been increasing. It is common belief that A2 and A4 pulleys are important in preventing bowstringing of the tendon which is associated with loss of flexion, flexion contracture and altering the kinematics of the tendon. We present a 24 -year-old male who underwent reconstruction of his Left index finger A2 pulley using allograft. The patient sustained a zone II flexor tendon injury to his left index finger. He had a complete laceration of his FDS and FDP tendons, and underwent repair of both. Postoperatively he regained full finger active and passive range of motion. However, 4 months postoperatively he suffered a rupture of his A2 pulley with bowstringing. Based on his age, postoperative course and presentation, and discussion of different treatment options. His A2 pulley was reconstructed with dermal allograft (Flex HD Structural-2 $\mathrm{cm} \times 4 \mathrm{~cm} \times 0.3 \mathrm{~mm}$ ). The allograft was passed through a bone tunnel made within the midportion of proximal phalanx. The allograft was wrapped in a one and a half loop fashion encircling only the flexor tendons and avoiding the extensor mechanism and neurovascular bundles. The patient is currently doing well and the reconstruction of the A2 pulley is still intact. Will start occupational therapy soon. Flexor tendon pulley injuries and ruptures can lead to pain, decreased range of motion, loss of strength, bowstringing and fixed flexion contractures. Although different methods have been described with success, each method has potential drawbacks. 


\title{
14. Predictors of complications following breast reduction surgery: A National Surgical Quality Improvement Program study of 16,812 cases
}

\section{Daniel P. Donato, Andrew M. Simpson, Alvin C. Kwok, Jayant P. Agarwal}

University of Utah

Aim: Breast reduction is one of the most common procedures performed by plastic surgeons. Despite good outcomes and high patient satisfaction, there is little national data examining the predictors leading to complications in this patient population. We accessed a national outcomes database to examine these factors.

Methods: This is a retrospective study examining the National Surgical Quality Improvement Program database from 2006 through 2015. Patients undergoing primary breast reduction were identified. Patients undergoing any cancer-related procedures were excluded. We identified patient-related and procedure related factors for analysis. Univariate and multivariate logistic regression analysis were used to identify independent predictors of complications.

Results: In total16,812 individual cases were identified. The overall complication rate for the cohort was $6.2 \%$ and the major complication rate was 3.0\%. Diabetes, bleeding disorder, hypertension, obesity, smoking, steroid use and prolonged operative time were associated with increased risk of complications $(P<0.05)$. Concurrent body contouring was a predictor of increased major complications, however liposuction was not.

Conclusion: Common surgical risk factors are associated with complications in breast reduction surgery. Although liposuction is not an independent risk factor, concurrent body contouring is associated with increased complications. Surgeons should be aware of these associations when discussing breast reduction with patients.

\section{Minimizing length of stay, narcotic use, operative times and complications combining an ERAS protocol to a two team approach in microsurgical breast reconstruction}

\author{
Harry Salinas, Miguel Medina, Elizabeth Hunter \\ Miami Cancer Institute - Baptist Health South Florida
}

Aim: Enhanced recovery after surgery (ERAS) pathways are being adopted to shorten postoperative length of stay (LOS) and decrease narcotic use. A two-team approach in microsurgical breast reconstruction has been shown to decrease operative times and complications. The authors sought to compare outcomes in microsurgical breast reconstruction using a two-team approach before and after the institution of an ERAS protocol.

Methods: Retrospective review of 44 consecutive patients undergoing free DIEP reconstruction with a continuous two team approach undergoing DIEP flap breast reconstruction. Twenty-one patients had an ERAS protocol consisting of intraoperative TAP block, intraoperative Ketorolac that is continued on a standing basis for $48 \mathrm{~h}$ and Q6h scheduled Tylenol. 
Results: The cohort was composed of 23 and 21 consecutive patients who underwent DIEP flap breast reconstruction before and after the institution of an ERAS protocol respectively. A total of 73 flaps were performed ( 37 and 36 flaps before and after ERAS respectively). LOS decreased from $4.82 \pm 0.77$ to 3 \pm 0 after the institution of the ERAS protocol. Of the ERAS patients, $42 \%$ did not take narcotics after discharge; the remainder of the group was on narcotics for 5.3 days on average (range 2-14 days). Operative times were on average 4.67 and $7.4 \mathrm{~h}$ for unilateral and bilateral procedures. Complications were similar in both cohorts.

Conclusion: The addition of an ERAS protocol to a two-team approach leads to a significant decrease in LOS and minimizes postoperative narcotic use after microsurgical breast reconstruction.

\section{An evaluation of infection related readmissions after breast reconstructive surgery using the Nationwide Readmissions Database}

Willem Collier, Melody Scheefer, Jaewhan Kim, Alvin Kwok

\section{University of Utah}

Aim: Hospital readmissions are costly. Thirty-day postoperative readmission rates are a common quality metric with associated financial consequences. Little is known about readmission rates for infection after implant-based breast reconstruction. We used the Nationwide Readmissions Database (NRD) to determine the rate and predictors of early and late hospital readmissions associated with infection after implant-based breast reconstruction.

Methods: Using the 2013-2014 NRD, we identified breast cancer patients undergoing implant-based breast reconstruction who had an infectious readmission with ICD-9 diagnosis and procedure codes. We used univariate and multivariate logistic regression models to identify patient demographic, comorbidity, and hospital predictors of infectious readmission within the early (0-30 day) and late (31-90 day) postoperative time-periods.

Results: In a weighted sample of the NRD, we identified 18,338 patients who underwent implant-based breast reconstruction. The overall infectious readmission rate for this group was $5.3 \%$. Only $38.4 \%$ of such readmissions occurred within the initial 30 days after surgery, and 39.5\% occurred 31-90 days after surgery. Medicaid patients (OR 1.45, $P=0.035$ ), median annual household income $<\$ 40,000$ (OR 1.41, $P=0.023)$, diabetes ( $\mathrm{OR} 1.52, P=0.030)$, and obesity ( $\mathrm{OR} 1.54, P=0.021)$ were independent predictors of overall infectious readmission. Only diabetes (OR 1.74, $P=0.041$ ) was an independent predictor of early infectious readmissions. Medicaid (OR 1.74, $P=0.033$ ), median annual household income $<\$ 40,000$ (OR $1.66, P=0.030$ ), obesity (OR 1.94, $P=0.007$ ), and length of hospital stay during the index procedure (OR 1.09, $P=0.028)$ were independent predictors of late infectious readmission.

Conclusion: Readmissions for infectious reasons after implant-based breast reconstruction occur more frequently beyond the initial 30-day postoperative period. Traditional thirty-day readmission rates may not be an adequate quality metric for breast reconstruction given the number of late postoperative readmissions. Early and late infectious readmissions have different predictors. Interventions targeting these predictors may decrease the number of readmissions. 


\section{Predicting success in breast augmentation: sizes, shapes and subjectivity}

\section{Hunter Moyer}

Regional Health, Rapid City

Aim: It remains difficult to define what women desire for breast augmentation. Accepted re-operation rates after primary breast augmentation remain over $20 \%$ in large studies, and implant exchange is one of the leading causes.

Methods: This study is a computer-generated, visual survey of eight female models with Vectra simulated augmentations using varying sizes of round and anatomic implants. A total of 314 females and 137 males rated the augmentations from best to worst for a total 1967 individual ratings.

Results: Natural, conservative and full are words associated positively with a successful breast augmentation. A review of current literature estimates the average breast implant volume in the United States between 350 and $374 \mathrm{~mL}$. In this study, the highest rated implant was a $457 \mathrm{~mL}$ round device. A normal distribution simulating the best ratings has an average of $416 \mathrm{~mL}$ (standard deviation $=48 \mathrm{~mL})(P=0.00000001$ vs. current literature average augmentation volume). Round implants were rated better than anatomic over the entire volume range (3.47 vs. 3.54, $P=0.00002)$, and within the central portion of higher rated volumes (2.67 vs. 3.01, $P=1.6 \mathrm{e}-12)$. There was no difference in preference of shape or volume between men and women or between respondents of varying ages, socioeconomic status or geographic location.

Conclusion: While patients describe the ideal breast augmentation as natural and conservative, they universally choose an implant that is $20 \%$ greater than bio-dimensional planning. Round implants were rated better, and there is no difference in preference between men and women.

\section{The use of bilateral paraspinous muscle flaps and bilateral composite latissimus dorsi and gluteus maximus flaps for closure of lumbosacral myelomeningocele defects in infants}

\section{Kathleen Holoyda, John Kestle, Barbu Gociman, Faizi Siddiqi}

\section{University of Utah}

Aim: Robust, reliable and reproducible closure of lumbosacral myelomeningocele defects remains a challenge. Closure of spinal defects following neurosurgical procedures with well-vascularized flaps in highrisk patients has been shown to reduce complications in the adult population. In infants with lumbosacral myelomeningocele, in addition to the relatively standard neurosurgical repair that consists of placode tubularization and dural repair, multiple methods of soft tissue coverage have been described. These include various cutaneous, fascial and muscle flaps and grafts. We present here our closure technique with wellvascularized flaps following lumbosacral myelomeningocele repair.

Methods: After the neurosurgical repair of lumbosacral myelomeningocele is completed bilateral composite latissimus dorsi muscleocutanous and gluteus maximus fasciocutanous flaps are elevated. The paraspinous muscle flaps are then elevated and medialized based on the lateral row arterial perforators to provide 
complete muscular coverage of the dural repair. The bilateral composite latissimus dorsi muscleocutanous and gluteus maximus fasciocutanous flaps are medialized and closed over the paraspinous muscle flap repair. Demographic and outcomes data of 7 patients from June 2014 to present were retrospectively reviewed.

Results: Of the 7 patients that underwent the above technique for closure of myelomeningocele defects, there have been no episodes of dehiscence with a median follow-up of 51 weeks (7-161 weeks). One patient experienced an area of small, superficial skin necrosis requiring surgical excision and reclosure.

Conclusion: Use of bilateral paraspinous muscle flaps and bilateral composite latissimus dorsi and gluteus maximus flaps provides robust coverage of lumbosacral defects following myelomeningocele repair in infants.

\title{
19. Outpatient bilateral mastectomies with immediate pre-pectoral breast reconstruction
}

\author{
Sara L. Struve, Barbara A. Pockaj, Raman C. Mahabir
}

Mayo Clinic Phoenix, Arizona

In the setting of breast cancer, ductal carcinoma in-situ, and other high risk patients, breast reconstruction can be offered in the immediate stage, at the same time as mastectomy. Immediate breast reconstruction has historically been done as an inpatient surgery, with at least one overnight stay in the hospital. Recently, we switched to performing these procedures in the outpatient setting. This case series documents ten bilateral mastectomies with immediate pre-pectoral direct-to-implant breast reconstruction patients, who were discharged home on post-operative day zero. To be able to achieve this outcome in an outpatient setting, there were several aspects we implemented prior to the transition. These included: pre-operative education and counseling, intra-operative measures, post-operative measures, and a discussion of the expected postoperative outcomes with patients and their families. Patient education and counseling was most important for setting expectations during and after surgery. Intra-operative measures included: IV Tylenol, Toradol, and steroids, as well as Exparel rib blocks, field blocks, and pectoralis major muscle blocks. Post-operatively, the patients were given scheduled analgesics for the first two post-operative days, then switching to prn medications thereafter. These factors all contributed to patients and families feeling comfortable enough for the patient to be discharged home the same day as their procedure.

\section{Use of social media in plastic surgery resident recruitment. A perspective from the applicants}

\section{Lacey Pflibsen, Nicole Kurnik, Ashley Howarth, Anthony Smith, Shelley Noland}

Mayo Clinic - AZ

Aim: There has been plenty of publications looking at how residency programs use social media websites of applicants to help with ranking; however, no such study has looked at the reverse, how social media websites of residency programs recruit applicants. This study aimed to investigate if plastic surgery residency programs are using social media platforms in resident recruitment. 
Methods: We sent out an eight question survey (SurveyMonkey) that is distributed to integrated plastic surgery PGY-1 residents via contacting the program coordinators. The questions were aimed at investigating which platforms were being viewed (Facebook, Instagram, Twitter), what images and information was important, and how it influenced applicants (to interview, rankings, etc\&).

Results: Preliminary data shows that although 90\% of residents themselves have a personal Facebook (70\% Instagram, $40 \%$ Twitter); the majority did not look at the social media page of residency programs prior to choosing to interview or ranking ( $90 \%$ and $70 \%$ respectively). It appears $60 \%$ of those that responded did not have a social media page at their institution; however, interestingly $87 \%$ of the respondents felt that portraying pictures of residents was influential and others commented that seeing resident-attending interaction was important.

Conclusion: It appears at this time social media platforms do not influence resident recruitment. With many programs starting either department or residency specific social media platforms, it will be interesting to see if increased awareness of social media platforms of programs will increase the importance of social media in resident recruitment.

\section{The scroll suspension suture in open septorhinoplasty \\ Leland Webb, Kevin Kalwerisky, Craig Czyz, Scott McCusker \\ United States Air Force}

Aim: The scroll area between the upper and lower lateral cartilages is often neglected during septorhinoplasty. A simple suture technique to address this area is presented as an adjunct to existing septorhinoplasty methods for improvement of form and function.

Methods: A single fine prolene suture is placed between the lower lateral cartilage and the soft tissue of the scroll area. By altering the vector of this suture, the tip position can be readily controlled, as can the shape of the lateral nasal wall.

Results: Thirty-six consecutive septorhinoplasty surgeries by a single surgeon were analyzed, and the scroll suspension suture was used in twenty-eight. All patients reported excellent subjective postoperative nasal breathing and revision surgery was performed in one, for reasons unrelated to the scroll suspension suture.

Conclusion: The scroll suspension suture is a useful technique to add to the rhinoplasty surgeon's toolbox and has positive aesthetic and functional characteristics.

\section{Successful reconstruction of bilateral oral commissure fusion post Stevens Johnson syndrome}

Dino Maglic, Ray Hosein, Barbu Gociman, Neal Moores, Shadia Flores, Faizi Siddiqi

University of Utah Health Care 
Aim: Stevens Johnson Syndrome (SJS) is a type IV hypersensitivity reaction commonly triggered by drugs which results in the uncontrolled destruction of keratinocytes with both cutaneous and mucosal involvement. A rare and often life threatening disease, SJS has an incidence of approximately 1 to 2 cases per 1 million. When involvement of the skin surpasses 10\% of the body surface area, it is designated as Stevens Johnson/ Toxic Epidermal Necrolysis Syndrome (SJS/TEN). A 19-year-old Hispanic female presented to the pediatric plastic surgery clinic with severe microstomia caused by bilateral oral commissure fusion post SJS/TEN after taking sulfamethoxazole/trimethoprim for the treatment of severe chronic acne vulgaris. Here, we report her successful reconstruction.

Methods: Triangular scar excision and mucosal Y-V advancement commissuroplasty were performed bilaterally under general anesthesia. The incisions were carefully design to avoid overlapping suture lines during the healing process.

Results: Our reconstruction commissuroplasty using triangular scar excision and mucosal Y-V advancement successfully resulted in normalization of the oral opening, and recreation of an esthetically pleasant mouth contour.

Conclusion: Although non-life threatening, microstomia can limit functionality and cause undue stress to the patients and their families. Triangular scar excision and mucosal Y-V advancement commissuroplasty proved to be an effective treatment modality of microstomia secondary to SJS/TEN.

\title{
23. Indications and outcomes of single-pedicle versus two-pedicle and multiple simultaneous thigh free flaps in head and neck reconstruction
}

\author{
Becky B. T. King, Ivan E. Rodriguez, Frederic W.B. Deleyiannis \\ University of Colorado
}

Aim: The anterolateral thigh (ALT) free flap is one of the most commonly used flaps for head and neck reconstruction. Given that perforators of an ALT flap routinely arise sequentially from the descending branch of the lateral circumflex artery as it descends down the thigh, a long ALT flap can be more reliably harvested than a wide ALT flap. The purpose of this study is to demonstrate indications and outcomes for single-pedicle ALT free flaps compared to double-pedicle ALT flaps as well as multiple simultaneous thigh (MST) flaps.

Methods: Our series of 81 consecutive patients undergoing head and neck reconstruction with an ALT flap was retrospectively reviewed. Receiver operating characteristics curve analysis was performed to determine our cut-off values for width and length of single-pedicle versus double-pedicle ALT flaps.

Results: Fifty-seven and 18 patients were reconstructed with an ALT flap with one or two pedicles, respectively. Six patients underwent MST flaps. Defect size (width $12 \mathrm{~cm}$, length $17 \mathrm{~cm}$ ) for cutaneous defects $(P<0.05)$, the presence of divergent mucosal defects, and through-and-through oral cavity or pharyngeal defects were associated with the use of two pedicles. While operative time was increased for the groups of double-pedicle ALT flaps and MST flaps, there were no flap complications including partial flap loss, venous congestion, or wound healing issues from poor flap perfusion.

Conclusion: Harvesting an ALT flap with two pedicles has the potential to reduce flap complications and should be considered for divergent and extremely wide or long defects. 


\title{
24. Combined TUG PAP using SPY as an aid in perineum reconstruction
}

\section{Karen Lo, David Mathes, Tae Chong}

\section{University of Colorado}

In perineal reconstruction, the goals are to provide coverage and eliminated dead space in an area prone to wound problems. The VRAM flap has traditionally been used due to its bulk and ease of transfer at the time as the oncologic surgery. However, when the abdomen is not available, other flaps are considered. We present the case of perineal reconstruction using combined TUG and PAP, with SPY angiography as an aid. KM a 59 yo $\mathrm{F}$ with h/o stage $3 \mathrm{c}$ invasive rectal adenocarcinoma s/p neoadjuvant chemoradiation. We planned a VRAM at the time of oncologic resection. However, she had positive margins; thus, underwent an APR, omental flap, mesh placement, hysterectomy and partial vaginectomy by her cancer surgeons. They placed a WV stating they did not want flap closure. A week later we were re-consulted for flap closure. As the VRAM was no longer an option, we decided on a thigh based flap. A doppler was used to identify the locations of the perforator for the TUG, ascending branch of the medial circumflex femoral artery, and the PAP, profunda artery. The PAP was clamped and we used SPY angiography to evaluate the perfusion based on the TUG alone. We noted poor perfusion of the distal skin paddle, which we intended to use to recreate the vagina. Under SPY, we released the clamp and saw immediate perfusion of the distal skin paddle and thus used both the PAP and the TUG in the reconstruction. The pt was discharged home without issues.

\section{Delayed DIEP flap loss: a complication of microvascular progress and earlier discharge}

\author{
Alicia Heelan Gladden, Becky B. T. King, Alexandra Kovar, Kristen Ohe, Colleen Murphy, Joyce \\ Aycock, David Mathes, Tae Chong
}

University of Colorado

\begin{abstract}
Aim: Traditional teaching is that microvascular complications most frequently occur in the early postoperative period, typically within $48 \mathrm{~h}$. The purpose of this study was to investigate deep inferior epigastric perforator (DIEP) total flap loss at our institution.
\end{abstract}

Methods: A retrospective analysis of patients who underwent DIEP flap breast reconstruction at a single institution was performed. Pre-operative demographic data and post-operative complications were recorded. Delayed flap loss was defined as non-salvageable flap presenting greater than $48 \mathrm{~h}$ after surgery.

Results: Eighty-eight patients underwent 137 DIEP flaps during the study period. Five patients (3.6\%) had threatened flaps in the first $48 \mathrm{~h}$ post-operatively and three of these were salvaged with emergent operative intervention. Five patients suffered total flap loss (3.6\%). Sixty percent (3/5) of flap losses occurred after patient discharge, with all three patients returning the day after discharge with a non-salvageable flap. When patients with early microvascular complications were compared to the delayed flap loss group, there were no significant differences in age, BMI, smoking status, diagnosis of diabetes or hypertension, radiation, or timing of reconstruction (all $P>0.05$ ).

Conclusion: Over half of our institutions flap losses were delayed, occurring after discharge. This finding contradicts the notion that most flap losses occur in the immediate post-operative period. The advancement of microsurgical techniques is reducing the frequency of flap loss during this early period, when flaps are 
closely monitored and prompt revision is possible. With greater emphasis on early patient discharge, perhaps more detailed patient education on return precautions is indicated.

\title{
26. Pedicled latissiumus dorsi myocutaneous flap for posterior thoracic reconstruction following eloesser flap takedown: a case series
}

\section{David Hill}

\author{
Rush University Medical Center
}

Pedicled latissimus flaps as the basis for thoracic reconstruction have been utilized for more than a century. While Dr. Abrashanoff described use of the flap as treatment for complicated bronchopleural fistula in 1911, its use in remedying iatrogenic chest wall defects resulting from external drainage procedures is less well-documented. Once medically stabilized, patients surviving chronic empyemas with Eloesser Flaps require reconstruction of the associated thoracic defect to restore quality of life, which may become of paramount importance. As the largest extrathoracic muscle flap which may be harvested on a single vascular pedicle, the latissimus dorsi serves as an ideal myocutaneous flap for obliterating Eloesser Flap defects. Its broad surface area and arc of rotation lend to coverage with minimal tension while avoiding exacerbation of respiratory mechanics. Although the latissimus dorsi muscle extends, adducts, and internally rotates the humerus, there are a number of other shoulder girdle muscles which combine to serve redundant functions, allowing for the muscle to be sacrificed with minimal loss of strength or form. We present a case series of pedicled latissimus dorsi myocutaneous flaps utilized for Eloesser Flap reconstruction with excellent aesthetic and functional results.

\section{Indocyanine green lymphangiography optimizes the identification and management of lymphatic leaks in the groin}

William Casey, Alanna Rebecca, Raman Mahabir, Lacey Pflibsen, Nadine Hillberg, Claire Jensen

Mayo Clinic - AZ

Aim: The treatment of lymphatic leaks and lymphoceles in the groin can be quite challenging with no optimal management determined to date. We postulate that ICG lymphangiography improves visualization of the site of a lymphatic leak and can optimize its management.

Methods: A retrospective review was conducted of all cases in which ICG lymphangiography was used in the management of lymphatic leaks in the groin over an 18-month span. The inciting surgical procedure resulting in the leak was determined. Following thorough debridement, ICG was injected intradermally in the distal extremity and the site of the lymphatic leak was documented (superficial or deep) and oversewn. Outcomes were reported with regards to healing, infection, time to drain removal, and adjunctive procedures.

Results: Fifteen patients underwent ICG lymphangiography during the surgical treatment of a lymphatic leak in the groin during the study period. In all cases, the site of the lymphatic leak was accurately identified and oversewn. In 8 cases, the site of the lymphatic leak was in the subcutaneous tissue superficial to the femoral vessels rather than medial to the femoral vessels in the area of the lymph node basin. A local muscle flap was used in 10 cases simultaneously. All wounds healed primarily without an associated wound or infection. 
Conclusion: ICG lymphangiography facilitated the identification of lymphatic leaks in the groin and optimized their management in these challenging cases, many of which may have been missed if the area around the inguinal lymph node basin was treated exclusively.

\section{Geometry of wound epithelialization}

\section{Michael Gordon}

University of Colorado

A mathematical computer model was created to test simple aspects of wound epithelization. Assumptions of the model included constant production of growth factors from the edge of the wound that diffused across the open area with a $1 / \mathrm{r} 2$ gradient from the cells at the edge of the wound. New cell growth was determined to occur when an adequate amount of growth factor had accumulated at a point in the open wound area. This model was then able to predict various characteristics of wound closure: speed of wound closure, effect of size of the wound on wound closure, shape of wound closure, effect of debridements on wound closure. These predictions were then tested in the laboratory setting using epithelial cell growth in a petri dish. Excellent agreement between the mathematical model and the laboratory model was noted. Even the often-noted, but mysterious, slowing down of wound healing as it approaches closure was observed and explained mathematically.

\section{Lumbar artery perforator flap breast reconstruction: achieving good aesthetic results at both donor and recipient sites}

\section{David Greenspun}

\section{Greenwich Hospital, Greenwich, Connecticut}

The lower abdominal wall is the most commonly used donor site for autologous breast reconstruction. The thighs and gluteal region are established alternative donor sites for breast reconstruction (PAP, TUG, GAP flaps) when the abdominal wall does not provide sufficient tissue or is otherwise unsuitable as a result of prior surgery. Flaps harvested from the buttock or thigh can produce good cosmetic results for the reconstructed breast, but this is frequently at the expense of unfavorable contour at the donor site. Harvest of the aforementioned flaps tends to flatten, or make concave, naturally convex surfaces of the body, thus producing unsatisfactory changes at their respective donor sites. In contradistinction, harvest of lumbar artery perforator (LAP) flaps, slightly superior to the iliac crest, accentuates the normal lordotic curvature of the lower back, and therefore produces favorable changes to the donor site contour whilst yielding excellent tissue for breast reconstruction. Harvest of the LAP flap pedicle is technically demanding and carries high stakes for donor site morbidity owing to the proximity of the spinal nerves to the vascular pedicle, however, these flaps can be performed safely and reliably. The routine use of arterial and venous grafts facilitates safe and efficient flap harvest as well as recipient site microsurgery and flap insetting. A retrospective series will be presented. With increased experience and good aesthetic results at both the donor and recipient sites, the LAP flap has emerged as an excellent second-choice flap for breast reconstruction when the abdomen is not suitable. 